\title{
miRNA-16 as a predictive factor for intracranial aneurysms in autosomal dominant polycystic kidney disease
}

\author{
Andrzej Kulesza, Agnieszka Kulesza, Magda Fliszkiewicz, Anna Łabuś, Leszek Pączek, \\ Mariusz Niemczyk \\ Department of Immunology, Transplant Medicine and Internal Diseases, Medical University of Warsaw, Poland
}

\begin{abstract}
Introduction. Autosomal dominant polycystic kidney disease (ADPKD) is the most common genetic renal disorder. It leads to multiple extra-renal complications, with intracranial aneurysms (IA) among the most serious. Biological markers could become tools in identifying patients at risk of an IA. MicroRNAs 16 (miR-16) and 25 (miR-25) have been proposed as being markers of IAs in the general population. In the current study, we attempted to discover if they may also be considered markers of IAs in ADPKD.

Material and methods. 64 renal transplant recipients with ADPKD were included. After magnetic resonance angiography of the brain, they were divided into a case group $(I A+, n=13)$ and a control group $(I A-, n=51)$. Expression of miRNAs in plasma was analysed by qRT-PCR.

Results. The expression of miR-16 was higher in the control (IA-) group. There was no statistically significant difference between the groups in terms of miR-25 expression.

Conclusions and clinical implications. MicroRNA-16 is a potential marker of IAs in renal transplant recipients with ADPKD. It may become a tool to identify patients who should undergo screening for an IA.

Key words: autosomal dominant polycystic kidney disease, biomarkers, intracranial aneurysm, kidney transplantation, micro RNA (Neurol Neurochir Pol 2021; 55 (3): 306-309)
\end{abstract}

\section{Introduction}

Autosomal dominant polycystic kidney disease (ADPKD) is the most common genetic renal disorder. Apart from endstage kidney disease (ESKD) in a large proportion of patients, it leads to multiple extra-renal complications; subarachnoid haemorrhages due to the rupture of an intracranial aneurysm (IA) are among the most serious [1]. In fact, the prevalence of intracranial aneurysms in ADPKD patients is approximately five times greater than in the general population, amounting to $10-11.5 \%[2,3]$, and may be further increased in special subpopulations e.g. renal transplant recipients with $\mathrm{AD}$ PKD [4]. Despite the fact that universal screening for IAs in ADPKD populations has been proposed [5, 6], it is still not widely accepted $[1,7]$. Therefore, there is a need to improve the selection of patients who should undergo screening to enable the detection of the maximum number of IAs in the pre-symptomatic period. Biological markers could become tools to identify patients at risk of an IA.

MicroRNAs (miRNAs) are short molecules of RNA (18-25 nucleotides in length). They are involved in post-transcriptional regulation of gene expression. They are present in plasma, and are relatively resistant to enzymatic degradation. Due to the unique patterns of change in different diseases, miRNAs are considered to be disease-specific biomarkers. MicroRNAs 16 (miR-16) and 25 (miR-25) have been proposed as being markers of IAs in the general population [8]. In the current study, we attempted to discover if they may also be considered markers of IAs in ADPKD.

\section{Material and methods}

Our work was a sub-study of a cross-sectional study on the prevalence of IAs in renal transplant recipients with ADPKD;

Address for correspondence: Mariusz Niemczyk, Department of Immunology, Transplant Medicine and Internal Diseases, Medical University of Warsaw, Nowogrodzka 59 Str., 02-006 Warsaw, Poland, e-mail: mniemczyk@wum.edu.pl 
its design and results have already been reported [9]. Participants in that study who consented to also participate in this sub-study were included. Participants were renal transplant recipients with ADPKD managed at the outpatient department of the Department of Immunology, Transplant Medicine and Internal Diseases of the Medical University of Warsaw, Poland. The inclusion criteria were: a diagnosis of ADPKD, being a renal transplant recipient, age 18-plus, lack of contraindications for magnetic resonance imaging, and written informed consent. Sixty-four patients met the inclusion criteria and were enrolled. In all patients, a 3D time-of-flight magnetic resonance angiography (MRA) of the brain was performed. All imaging studies were performed between January 2015 and November 2019 using an Ingenia 1.5T HP (Philips Healthcare, Best, the Netherlands) scanner, and in total $20 \mathrm{ml}$ of blood was collected for further examination. Peripheral venous blood was collected into EDTA-containing tubes. Whole blood was first centrifuged at $1,600 \mathrm{~g}$ for 10 minutes, and then the supernatant was transferred into a fresh tube and centrifuged again at $13,000 \mathrm{~g}$ for 10 minutes. The clear plasma was aliquoted and stored at $-80^{\circ} \mathrm{C}$ until use. All procedures were carried out on ice.

Then, according to the design of a case-control study, the patients were divided into two groups according to the presence or absence of IA.

Expression of miRNA-16 and miRNA-25 was analysed by qRT-PCR. A column method miRNeasy Serum/Plasma advanced kit (Quiagen) was used for RNA isolation from previously frozen plasma samples according to the manufacturer's instructions. RNA quality and quantity were determined spectrophotometrically with a NanoDrop ND-2000 (NanoDrop Technologies, Inc.). A Quiagen Spike-In control C.elegans miR39 miRNA mimic was used as an internal control for miRNA expression. Specific TaqMan Gene Expression assays were purchased from Applied Biosystems. A TaqMan MicroRNA reverse transcription kit and Universal Master Mix II (Applied Biosystems) were used for RNA reverse transcription and real-time PCR amplification. RT-PCR was performed on an ABI Prism 7500 Sequence Detection System (Applied Biosystems) using specific TaqMan primers and probes (Applied Biosystems): hsa-miR-16 (UAGCAGCACGUAAAUAUUGGCG) and hsa-miR-25 (AGGCGGAGACUGGGCAAUUG). U6 was used as a housekeeping gene. Each sample was analysed in triplicate. The relative gene expression was calculated by the
$2^{-\Delta \Delta C t}$ method. The results were presented as a fold change of gene expression in patients with an intracranial aneurysm and in those without, where the reference point was expression in the non-aneurysmal group.

Normality of data distribution was measured with a Kolmogorov-Smirnov test. Statistical analysis was performed by comparing $\Delta \mathrm{Ct}$ values using nonparametric tests for independent samples (Mann-Whitney U test). All analyses were performed with Statistica software version 12.5 (StatSoft, Tulsa, OK, USA). A $P$ value of $<.05$ was considered statistically significant.

This study was conducted in accordance with the principles of the Declaration of Helsinki, and the ethics committee of the Medical University of Warsaw approved the protocol. All patients gave written informed consent for inclusion in the study.

\section{Results}

64 patients were included in the study, comprising $33(52 \%)$ men and 31 (48\%) women. All included patients were white and all were recipients of their first renal transplant. The characteristics of the study group are set out in Table 1 . Among this study group, IA was detected in 13 cases, while 51 patients were IA-free.

The expression of miR-25 was found in all patients, and the expression of miR-16 was found in all but one patient; that case was excluded from analysis. We observed statistically significant differences in the expression of miR-16 between patients with and without IA, based on the accepted level of significance $p<0.05$ and $Z$ statistic of the Mann-Whitney $\mathrm{U}$ test $(\mathrm{Z}=2.90616)$ with continuity correction $\mathrm{p}=0.01658$ as well as based on accurate $U$ statistics (743). The expression of miR-16 was higher in the control (non-aneurysmal) group, and the ratio of mean miR-16 expression level was 1.00:0.39 (control:cases). A similar relationship was also observed for miR-25, although the results did not reach statistical significance $(Z=-0.1441 ; U=1,126.5 ; p=0.88866)$.

\section{Discussion}

The prevalence of IAs in renal transplant recipients with ADPKD has been assessed as being between 14.9\% [4] and $22.7 \%$ [9]. Developing biomarkers may shed light on the

Table 1. Characteristics of study group

\begin{tabular}{|c|c|c|c|}
\hline \multirow[t]{2}{*}{ Characteristic } & \multicolumn{3}{|c|}{ Value } \\
\hline & Whole group $(n=64)$ & IA (+) group $(n=13)$ & IA (-) group $(n=51)$ \\
\hline Men/women (n, \%) & $33 / 3152 \% / 48 \%$ & $6 / 746 \% / 54 \%$ & $27 / 2453 \% / 47 \%$ \\
\hline Median age, years (range, IQR) & $60.7(38-78,11.1)$ & $55.7(37.5-67.2,8.7)$ & $62.1(39.5-78.2,10.5)$ \\
\hline Median time after transplant in months (range, IQR) & $35.6(1-236,77.9)$ & $8.9(1.2-100,49.7)$ & $46(0.6-236.2,72.1)$ \\
\hline Median eGFR [CKD-EPI formula], $\mathrm{ml} / \mathrm{min} / 1.73 \mathrm{~m}^{2}$ (range, IQR) & $45.6(7-97.3,32.3)$ & $38.9(22.4-82.6,22.5)$ & $47.7(7-97.3,34.5)$ \\
\hline
\end{tabular}

IQR - interquartile range, eGFR - estimated glomerular fraction rate, IA - intracranial aneurysm 
pathogenesis of IAs in this group. Additionally, biomarkers may become tools useful in the selection of patients who should undergo imaging for an IA.

miR-16 belongs to the most abundant miRNAs present in human plasma. Because its level was considered to be relatively stable, it was previously used as the housekeeping gene in qPCR assays. However, it was later shown that the plasma levels of miR-16 can change in certain diseases. According to the study by Li et al. [8], expression of miR-16, together with miR-25, is increased in patients with IA compared to healthy controls. miR-16 is expressed by vascular endothelial cells and is involved in angiogenesis [8]. While the function of endothelial cells is disturbed in ADPKD [10], it seemed desirable to verify the results of Li et al. [8] in ADPKD patients.

Our results show that, indeed, plasma expression of miR16 is altered in ADPKD patients after renal transplantation with IA, but, in contrast to the results of Li et al. [8], we observed decreased expression. Additionally, we did not observe altered plasma levels of miR-25 in this particular group of patients. Therefore, according to our results, miR-16, but not miR-25, could become a potential biomarker of IAs in ADPKD patients after renal transplantation.

Our results suggest that the pathogenesis of IAs in ADPKD may be different compared to IAs in the general population. That may explain numerous differences in the clinical picture of IAs between ADPKD patients and the general population, namely their prevalence, age at development, lack of gender differences etc. [2]. Thus, there is a need to take into account the distinct clinical proceedings with these particular cases. For instance, clinical biomarkers of IAs, if developed for the general population, may not be applicable to ADPKD patients, at least after kidney transplantation. Additionally, it would be of interest to compare levels of miR-16 in renal transplant recipients to those in the general population. This could potentially explain the differences between our results and those of Li et al. [8].

The fact that the plasma levels of miR-16 may change in certain diseases could also impact upon our results. For example, blood levels of miR-16 can change during vascular rejection of a renal graft [11]. In all of our patients, the graft function was stable at the moment of sample collection; however, our results should be interpreted cautiously due to the fact that some confounding factors might be omitted in the analysis. Therefore, further studies on this subject are needed.

Some limitations of our study should be noted. Firstly, due to the characteristics of the Polish population, only Caucasians were included in our study; results in other populations may differ. Secondly, the group was relatively small, and our observations should be confirmed in a larger study including populations of different ethnicities. Thirdly, only renal graft recipients were included in this study, and additional factors connected to this fact (e.g. immunosuppressive treatment, asymptomatic infections) might lead to bias. Additionally, renal graft recipients are in general older than patients in the pre-ESKD period, and therefore our results may not reflect the situation in the entire ADPKD population.

The clinical utility of miR-16 as a biomarker of IA in renal transplant recipients with ADPKD, and, possibly, in the whole ADPKD population, requires verification in further studies.

\section{Conclusions and clinical implications}

MicroRNA-16 is a potential marker of IA in renal transplant recipients with ADPKD. It may become a tool to identify patients who should undergo screening for IAs.

Funding: This publication was prepared without any external source of funding.

Conflict of interest: The authors declare no conflict of interest.

\section{References}

1. Rangan GK, Alexander SI, Campbell KL, et al. KHA-CARI guideline recommendations for the diagnosis and management of autosomal dominant polycystic kidney disease. Nephrology. 2016; 21(8): 705-716, doi: 10.1111/nep.12658, indexed in Pubmed: 26511892.

2. Zhou Z, Xu Y, Delcourt C, et al. Is Regular Screening for Intracranial Aneurysm Necessary in Patients with Autosomal Dominant Polycystic Kidney Disease? A Systematic Review and Meta-analysis. Cerebrovasc Dis. 2017; 44(1-2): 75-82, doi: 10.1159/000476073, indexed in Pubmed: 28502970.

3. Cagnazzo F, Gambacciani C, Morganti R, et al. Intracranial aneurysms in patients with autosomal dominant polycystic kidney disease: prevalence, risk of rupture, and management. A systematic review. Acta Neurochir (Wien). 2017; 159(5): 811-821, doi: 10.1007/s00701017-3142-z, indexed in Pubmed: 28283868.

4. Kim JY, Jung SC, Ko Y, et al. Intracranial aneurysms in patients receiving kidney transplantation for autosomal dominant polycystic kidney disease. Acta Neurochir (Wien). 2019; 161(11): 23892396, doi: 10.1007/s00701-019-04060-7, indexed in Pubmed: 31502043.

5. Flahault A, Trystram D, Nataf F, et al. Screening for intracranial aneurysms in autosomal dominant polycystic kidney disease is cost-effective. Kidney Int. 2018; 93(3): 716-726, doi: 10.1016/j.kint.2017.08.016, indexed in Pubmed: 29061331.

6. Malhotra A, Wu X, Matouk CC, et al. MR Angiography Screening and Surveillance for Intracranial Aneurysms in Autosomal Dominant Polycystic Kidney Disease: A Cost-effectiveness Analysis. Radiology. 2019; 291(2): 400-408, doi: 10.1148/radiol.2019181399, indexed in Pubmed: 30777807.

7. Chapman AB, Devuyst O, Eckardt KU, et al. Conference Participants. Autosomal-dominant polycystic kidney disease (ADPKD): executive summary from a Kidney Disease: Improving Global Outcomes (KDIGO) Controversies Conference. Kidney Int. 2015; 88(1): 17-27, doi: 10.1038/ki.2015.59, indexed in Pubmed: 25786098.

8. Li P, Zhang Q, Wu X, et al. Circulating microRNAs serve as novel biological markers for intracranial aneurysms. J Am Heart Assoc. 2014; 3(5): e000972, doi: 10.1161/JAHA.114.000972, indexed in Pubmed: 25249297. 
9. Kulesza A, Gradzik M, Kulesza A, et al. Intracranial aneurysms in renal transplant recipients with autosomal dominant polycystic kidney disease. Pol Arch Intern Med. 2020; 130(12): 1111-1113, doi: 10.20452/pamw.15648, indexed in Pubmed: 33056941.

10. Bellos I, Kontzoglou K, Perrea DN. Markers of endothelial dysfunction and arterial stiffness in patients with early-stage autosomal dominant polycystic kidney disease: A meta-analysis. Int J Clin Pract.
2021; 75 (4): e13721, doi: 10.1111/ijcp.13721, indexed in Pubmed: 32946652 .

11. Matz M, Fabritius K, Lorkowski C, et al. Identification of T Cell-Mediated Vascular Rejection After Kidney Transplantation by the Combined Measurement of 5 Specific MicroRNAs in Blood. Transplantation. 2016; 100(4): 898-907, doi: 10.1097/TP.0000000000000873, indexed in Pubmed: 26444957. 